\title{
Crossed Immunoelectrophoresis and Enzyme-linked Immunosorbent Assay of the Cell-surface Antigens of Bacteroides fragilis
}

\author{
By GARY COUSLAND AND IAN R. POXTON* \\ Bacteriology Department, University of Edinburgh Medical School, Teviot Place, \\ Edinburgh EH8 9AG, UK
}

(Received 31 August 1983; revised 25 October 1983)

\begin{abstract}
Antisera were raised to whole, live cells of a reference strain (NCTC 9344) and two clinical isolates (GNAB 92 and GNAB 4) of Bacteroides fragilis. Each antiserum was reacted in crossed line immunoelectrophoresis (CLIE) with EDTA-heat-sonication-prepared outer membrane $(\mathrm{OM})$ complex from $10 \mathrm{~B}$. fragilis strains. In addition, the antisera were reacted with these antigens in an enzyme-linked immunosorbent assay (ELISA). In CLIE, the antisera raised to the reference strain and one of the clinical isolates (GNAB 92) demonstrated a heat labile antigen which was common to all 10 of the test strains. Lipopolysaccharide (LPS) prepared from both the clinical isolates produced three major precipitin lines when reacted with their homologous antisera in crossed immunoelectrophoresis (CIE). In both cases, these three antigens were present as major components of the OM complex. Each antiserum reacted significantly in ELISA with all test OM complex preparations. Inhibition of ELISA showed that carbohydrates were the predominant cross-reactive antigens in ELISA and that in the case of the clinical isolate GNAB 4, most of the cross-reactive antigenic activity was present in the homologous LPS preparation.
\end{abstract}

\section{INTRODUCTION}

Bacteroides fragilis is the most common anaerobic organism isolated from clinical specimens (Wren et al., 1977). It is a significant pathogen in various wound infections, especially those of the abdomen (Finegold, 1977). It can be found either in pure culture or more frequently together with facultative organisms. Bacteroides fragilis has surface components, usually referred to as capsule, which apparently make it more virulent than the more common faecal commensal bacteroides, e.g. B. vulgatus, to which it is closely related (Onderdonk et al., 1977). These observations, and the subsequent need for a rapid and more reliable sero-identification scheme, have led to several studies of the cell surface of the species.

Methods used to examine the surface antigens of $B$. fragilis have most often concentrated on heat stable carbohydrate components and have included tube agglutination of whole, boiled cells (Làmbe \& Moroz, 1976; Elhag \& Tabaqchali, 1978) and immunodiffusion of cell extracts (Abshire et al., 1979; Cherniak et al., 1979). The antigenic properties of purified lipopolysaccharide (LPS) have also been studied (Hofstad, 1975, 1977; Meizel-Mikolajczyk et al., 1981). All investigations have shown complex patterns of cross-reactions and thus considerable antigenic diversity associated with heat stable antigens.

The role of individual cell surface components as antigens is uncertain. Kasper \& Seiler (1975) and Kasper (1976) isolated a high molecular weight, species-specific capsular polysaccharide antigen, but Kasper $e t$ al. (1983) have reported that the purified substance was a mixture of LPS and capsule. Recent studies have also indicated that LPS purified from $B$. fragilis by the aqueous phenol method of Westphal \& Luderitz (1954) may be contaminated with non-O-antigenic, carbohydrate precipitating antigens (Hofstad, 1981, 1982).

Abbreviations: CIE, crossed immunoelectrophoresis; CLIE, crossed line immunoelectrophoresis; ELISA, enzyme-linked immunosorbent assay; OM, outer membrane. 
An enzyme-linked immunosorbent assay (ELISA) has been developed which detects speciesspecific antigens in B. fragilis outer membrane preparations (Poxton, 1979; Poxton et al., 1982). However, the relationship of the ELISA antigens to LPS, other carbohydrate antigens and outer membrane proteins is not known. In this study, the nature of the antigens detected by ELISA was investigated by the inhibition of ELISA technique. Crossed immunoelectrophoresis (CIE) was also performed to investigate the relationship between precipitating antigens and the antigens detected by ELISA.

\section{METHODS}

Culture of bacteria and preparation of EDTA-outer membrane complex. Bacteroides fragilis NCTC 9344 and nine laboratory isolates (GNAB 4, 85, 86, 87, 88, 90,92,97 and 98) that had been previously characterized according to the criteria of Duerden et al. (1980) were cultured anaerobically from freeze-dried inocula in PPY medium (Holbrook et al., 1977). Outer membrane (OM) complexes were prepared by the EDTA-heat-sonication treatment previously described (Poxton \& Brown, 1979), except that incubation with EDTA buffer was extended from $30 \mathrm{~min}$ to $2 \mathrm{~h}$ and all samples were concentrated in an ultrafiltration cell with a PM-10 membrane (Amicon Corp., Lexington, Mass., USA).

Lipopolysaccharide (LPS) preparation. LPS was extracted from freeze-dried bacteria that had been grown in PPY medium for $18 \mathrm{~h}$ by the aqueous phenol procedure developed by Westphal \& Luderitz (1954). It was purified and washed by centrifugation at 100000 for $3 \mathrm{~h}$.

Treatment of $O M$ complex. Separate samples of EDTA-released OM complex at a concentration of $0.5-1.0 \mathrm{mg}$ protein $\mathrm{ml}^{-1}$ were treated with: (a) Heat $\left(121^{\circ} \mathrm{C}\right)$ for $15 \mathrm{~min}$.; (b) $0 \cdot 1 \mathrm{M}$-sodium periodate in sodium acetate buffer (pH 5) at $20^{\circ} \mathrm{C}$ overnight. Excess periodate was consumed by the addition of ethylene glycol and samples were dialysed against $0.15 \mathrm{M}$-phosphate buffer pH 7.4 (PBS). A control sample was taken through each stage with the omission of sodium periodate. (c) A mixture of pronase (BDH) and trypsin (Sigma) both at a concentration of $0.1 \mathrm{mg} \mathrm{ml}-1$, overnight at $37^{\circ} \mathrm{C}$. A control sample was also incubated at $37^{\circ} \mathrm{C}$ without the enzymes.

Preparation of antisera. Antisera were raised against washed live cells of B. fragilis NCTC $9344, B$. fragilis GNAB 4 and B. fragilis GNAB 92 in New Zealand White rabbits as previously described (Poxton, 1979).

Enzyme-linked immunosorbent assay (ELISA). Indirect ELISA was carried out as previously described (Poxton \& Byrne, 1981). Briefly, OM complex was diluted to $30 \mu \mathrm{g}$ protein $\mathrm{ml}^{-1}$ in $\mathrm{pH} 9.6$ carbonate buffer and coated onto wells of microtitre plates. After reaction with rabbit antiserum, anti-rabbit-IgG-alkaline phosphatase conjugate (Miles Laboratories, Stoke Poges, Slough, UK) and substrate ( $p$-nitrophenyl phosphate, Sigma) results were read in a Titertek Multiskan (Organon Teknika, St Neots, Cambs., UK). The end-point of the titration (titre) was the first dilution of serum with an $A_{405}$ value of less than 1.6. An ELISA inhibition test was performed by preincubating antisera with potential inhibitors: antiserum at four or eight times its titre, depending on strength of heterologous reaction, was incubated with an equal volume of doubling dilutions of untreated, heat treated or periodate treated OM complex ( $2 \mathrm{mg}_{\mathrm{g}}$ protein $\left.\mathrm{ml}^{-1}\right)$ or LPS $\left(400 \mu \mathrm{g}\right.$ carbohydrate $\left.\mathrm{ml}^{-1}\right)$. A 1 in 2 dilution of OM complex or LPS was the first inhibitor concentration used. Absorbance $\left(A_{405}\right)$ readings for each inhibited reaction were expressed as a percentage of the normal uninhibited reaction.

Crossed immunoelectrophoresis (CIE) with intermediate gel. The procedure was essentially that described for CIE by Weeke (1973). OM complexes were adjusted to a concentration of 2 or $3 \mathrm{mg}$ protein $\mathrm{ml}^{-1}$ in PBS containing $1 \%$ (v/v) Triton X-100. These antigens $(16 \mu \mathrm{l})$ were examined against homologous antisera in CIE on sheets $(5 \times 5 \mathrm{~cm})$ of GelBond (Marine Colloids, Rockland, Maine, USA). Gel and electrophoresis buffer composition were as previously described (Poxton \& Byrne, 1981). Electrophoresis was at $12.5 \mathrm{~V} \mathrm{~cm}^{-1}$ for $1.5 \mathrm{~h}$ in the first dimension and at $12 \mathrm{~V} \mathrm{~cm}^{-1}$ for $16 \mathrm{~h}$ in the second dimension, both at $4^{\circ} \mathrm{C}$. Gels were washed, pressed and stained with Coomassie blue. A $5 \times 1 \mathrm{~cm}$ agarose strip (intermediate gel) containing antiserum (or nothing in controls) was incorporated between the first and second dimension gels.

Crossed line immunoelectrophoresis (CLIE). The conditions for CLIE were as described above for CIE except that the intermediate gel contained heterologous $O M$ antigen preparations ( $150-450 \mu g$ protein). This technique detects cross-reactions between the homologous antigens run in the first dimension and heterologous antigens contained in the intermediate gel (Krell, 1973).

Analytical techniques. Protein concentrations were estimated by the Lowry method, and carbohydrate as glucose equivalents was estimated by the method of Dubois et al. (1956).

\section{RESULTS}

CIE and CLIE

Crossed immunoelectrophoresis of OM complex from $B$. fragilis strains NCTC 9344 (reference strain), GNAB 92 and GNAB 4 (clinical isolates) was performed against homologous antisera. 

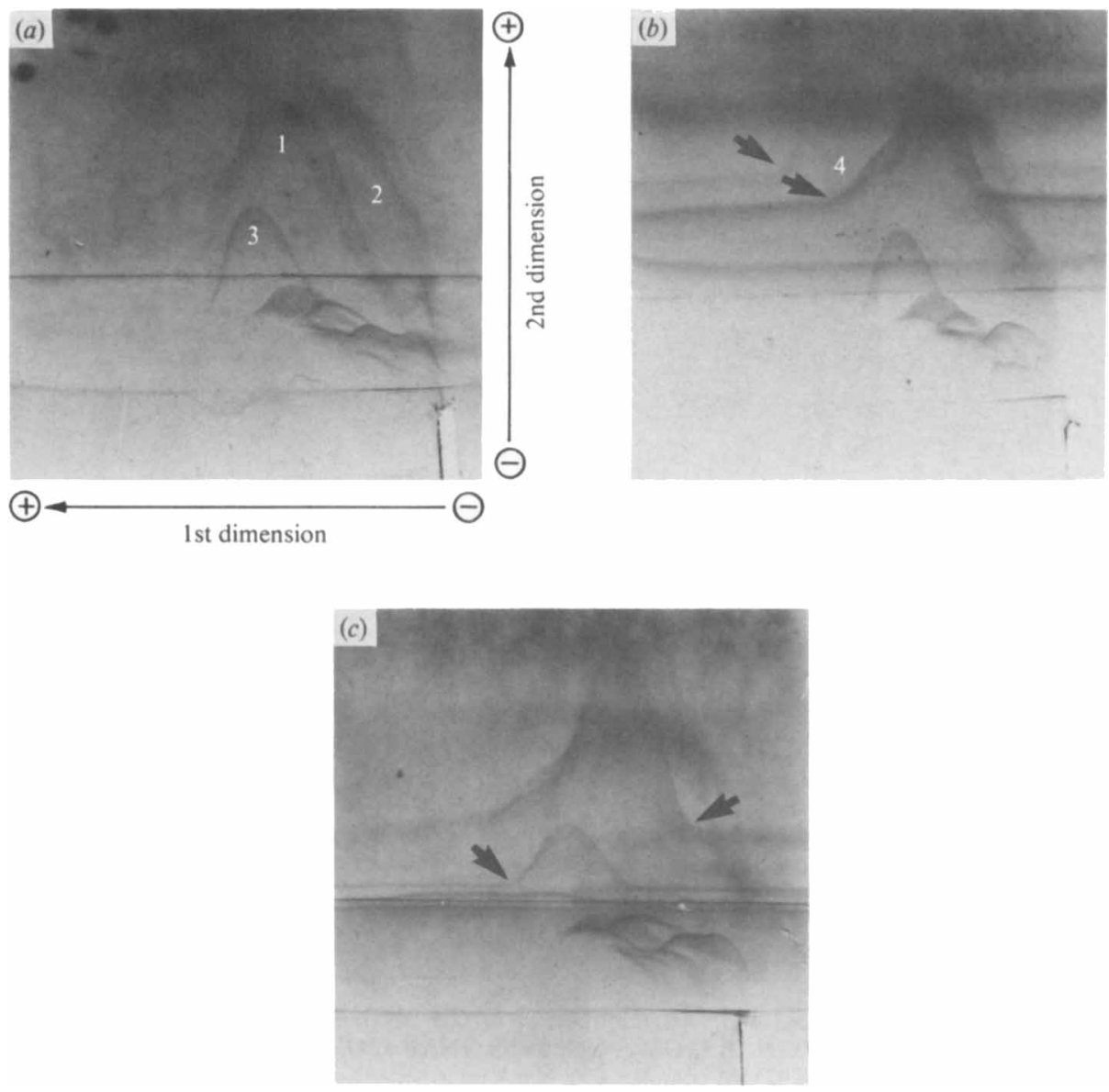

Fig. 1. CIE and CLIE reactions of 9344 antiserum: (a) CIE of 9344 OM complex with blank intermediate gel; (b) CLIE of 9344 OM complex with GNAB 85 OM complex in the intermediate gel; (c) as in (b) except with GNAB 90 OM complex in the intermediate gel. In the first dimension $16 \mu 1$ NCTC 9344 OM complex (48 $\mu$ g protein) was run, against $500 \mu$ antiserum in the second dimension. Intermediate gels $(b$ and $c$ ) contained $450 \mu \mathrm{g}$ protein in $0.75 \mathrm{ml}$ agarose gel. The numbers indicate the precipitin arcs of cross-reacting antigens.

The presence of cross-reacting precipitating antigens in heterologous strains was detected by inclusion of heterologous OM complex in an intermediate gel (CLIE).

Reactions with NCTC 9344 antiserum. The homologous reaction with NCTC 9344 OM complex produced several precipitin arcs (Fig. $1 a$ ), and those that were subsequently proved to be cross-reacting antigens are numbered. CLIE was done with OM antigens from the nine heterologous test strains. The results obtained with OM complex from GNAB 85 and GNAB 90 in intermediate gels are shown in Fig. $1(b, c)$. Appearance of a horizontal precipitin line indicated a reaction between the NCTC 9344 antiserum and a heterologous antigen, and the joining of such a line with a homologous precipitin arc indicated a cross-reaction between the antigens. Cross-reaction of the GNAB 85 OM complex with antigen 1 can be seen in Fig. $1(b)$ (arrowed). This strain also cross-reacted with a faint precipitate that was not visible in the control reaction but which became apparent in CLIE reactions (arrowed). This antigen was numbered 4 (Fig. $1 b$ ). In Fig. $1(c)$ it can be seen that the OM preparation from GNAB 90 crossreacted with antigens 1 and 3 (arrowed). The nine test strains varied in their cross-reactions with antigens 2, 3 and 4, but all strains possessed an antigen that cross-reacted with antigen 1, 

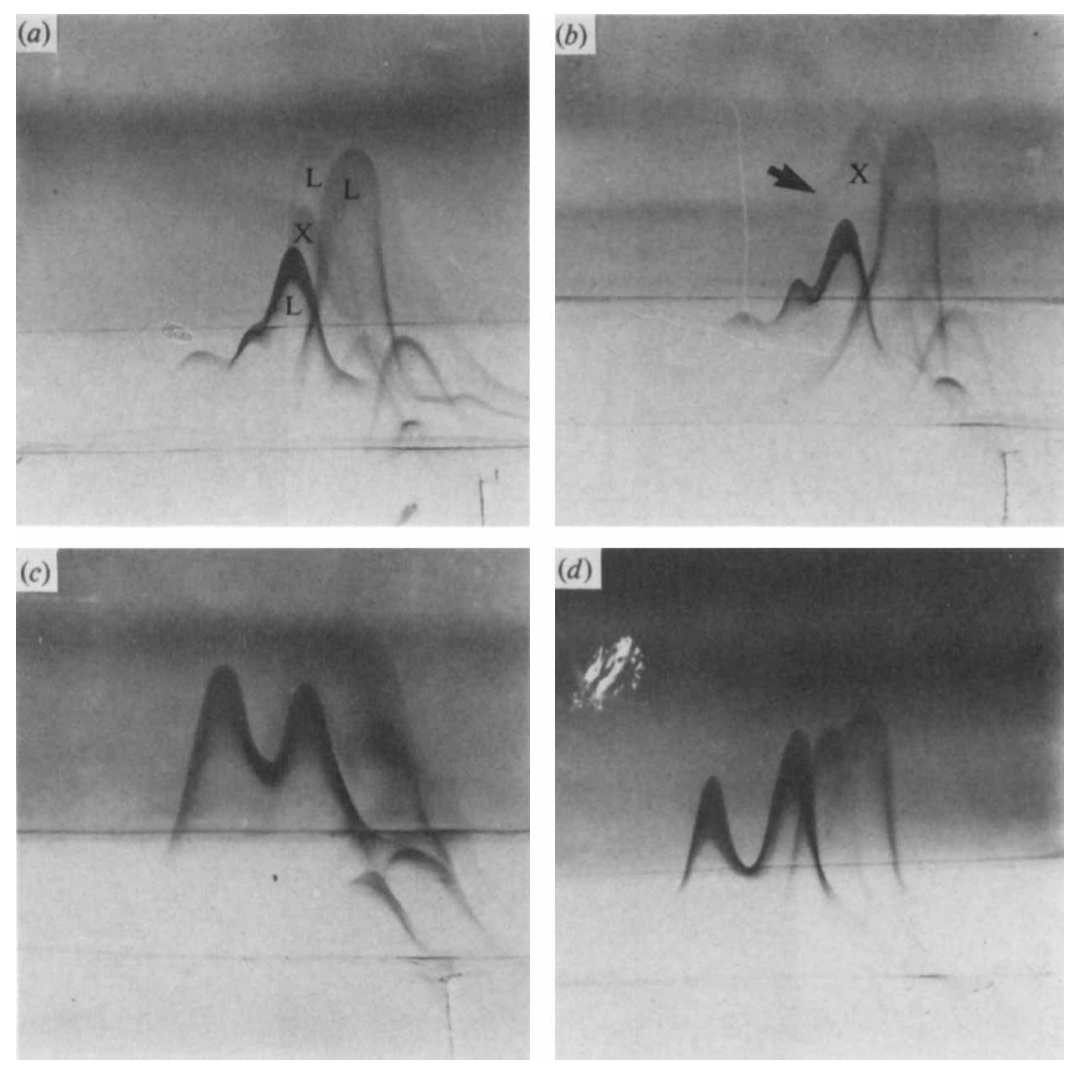

Fig. 2. CIE and CLIE reactions of GNAB 92 antiserum : (a) CIE of GNAB 92 OM complex with blank intermediate gel; (b) CLIE of GNAB 92 OM complex with GNAB 87 OM complex in the intermediate gel; (c) CIE of heated GNAB 92 OM complex with blank intermediate gel; (d) CIE of GNAB 92 LPS with blank intermediate gel. In the first dimension $16 \mu$ GNAB 92 OM complex (48 $\mu \mathrm{g}$ protein) or LPS ( $4 \mu \mathrm{g}$ carbohydrate) was run, against $375 \mu \mathrm{l}$ antiserum in the second dimension. Intermediate gel (b) contained $150 \mu g$ protein in $0.75 \mathrm{ml}$ agarose gel. $L$ and $X$ mark the cross-reacting antigens.

indicating the presence of a $B$. fragilis common antigen. Periodate treatment of the NCTC 9344 OM preparation had little effect on the homologous picture and the antigen 1 precipitate was unmodified. Heating the $\mathrm{OM}$ complex $\left(121^{\circ} \mathrm{C}\right.$ for $\left.15 \mathrm{~min}\right)$ destroyed most of the antigens although some ill-defined precipitates remained. It was unclear which of the antigens these faint reactions represented. The damage to antigen $I$ by heat and its resistance to periodate suggested that it may be a protein. When the $\mathrm{OM}$ complex was treated with pronase and trypsin, the antigen 1 precipitate became faint and cross-reacted poorly in CLIE indicating that antigen $l$ is a protein.

Purified NCTC 9344 LPS did not react strongly with the antiserum, but did produce faint precipitates representing at least two distinct antigens.

Reactions with GNAB 92 antiserum. The homologous reaction with GNAB 92 OM complex is shown in Fig. 2(a). Outer membrane complexes from the nine heterologous test strains were included in intermediate gels and an example of the CLIE pattern obtained with GNAB 87 is shown in Figure $2(b)$. A cross-reaction with the antigen marked $\mathrm{X}$ in Figure 2(a) occurred (arrowed). The antigen $X$ precipitate joined up with the horizontal precipitin line and was positioned higher in the gel than in the control. The OM antigens from the other eight heterologous strains also cross-reacted with antigen X. Most of the strains only showed crossreaction with this single antigen. Periodate treatment of the GNAB 92 OM complex destroyed all the major antigens, including antigen $\mathrm{X}$. Several antigens were resistant to heat (Fig. $2 c$ ), 
although antigen $\mathrm{X}$ was destroyed. This is difficult to see from Fig. 2(c), but the destruction of antigen $\mathrm{X}$ was confirmed by running a CLIE with untreated homologous antigen in the first dimension and heated heterologous OM complex in the intermediate gel (i.e. as Fig. $2 c$ but unheated): no line precipitate occurred. Treatment with pronase and trypsin hardly affected the picture, although the mobility of antigen $X$ towards the anode increased slightly.

The results indicated that there are several distinct carbohydrate antigens associated with the GNAB $92 \mathrm{OM}$ complex. Also, a heat labile, periodate sensitive common antigen (X), possibly a glycoprotein, exists. Purified GNAB 92 LPS produced three distinct antigens in CIE (Fig. $2 d$ ). When LPS was incorporated into the intermediate gel of the homologous OM complex reaction, it cross-reacted with the three antigens marked ' $L$ ' in Figure $2(a)$.

Reactions with GNAB 4 antiserum. The homologous reaction with GNAB 4 OM complex yielded several precipitates (Fig. $3 a$ ). In contrast to the results obtained with the NCTC 9344 and GNAB 92 antisera, CLIEs with GNAB 4 antiserum did not demonstrate a common antigen in the ten test strains. The OM complex from strain GNAB 86 cross-reacted with antigen A (Fig. 3b, arrowed) as did strain GNAB 97. Strains NCTC (Fig. 3c, arrowed) and GNAB 87 cross-reacted with antigen $C$ and appear to have cross-reacted weakly with antigen A. Strain GNAB 98 was the only strain to cross-react with antigen B. Cross-reactions of OM complexes from strains GNAB 85, GNAB 88, GNAB 90 and GNAB 92 could not be demonstrated by CLIE, even when levels of $600 \mu \mathrm{g}$ protein were included in the intermediate gel. Periodate treatment destroyed most of the antigenic activity of the GNAB 4 OM complex and at least three of the antigens were resistant to heat (Fig. $3 d$ ).

Purified GNAB 4 LPS was run against homologous antiserum (Fig. $3 e$ ): two heat resistant antigens were detected. The GNAB 4 OM complex was run against homologous antiserum with LPS in the intermediate gel. The CLIE pattern obtained confirmed the presence of antigens $A$ and $C$ in LPS but also detected two other antigens. One of these cross-reacted with antigen $B$ but the other showed no cross-reaction with the OM complex precipitates.

The results indicated that GNAB 4 antiserum reacted with several carbohydrate antigens in the homologous OM complex. Four precipitating antigens were detected in the LPS preparation, three of which were the major cross-reacting antigens. The presence of four precipitating antigens in ultracentrifuged LPS indicates co-purification of antigens other than LPS.

The antisera to NCTC 9344 and GNAB 92 had demonstrated a common antigen in the ten test strains (antigen 1 and antigen $\mathrm{X}$ ). They were both heat labile but their sensitivity to periodate differed. In an attempt to determine if they were in fact the same antigen, CIEs with antiserum-containing intermediate gels were run. Antigen from GNAB 92 was run through an intermediate gel containing GNAB 9344 antiserum into GNAB 92 antiserum (Fig. 4). When compared to the control CIE without antiserum in the intermediate gel (Fig. $2 a$ ) precipitin line $\mathrm{X}$ is seen to have precipitated in the intermediate gel. In the converse experiment, NCTC 9344 antigen run through GNAB 92 antiserum, a similar effect was seen with line 1 (result not shown due to difficulty in reproduction). This demonstrated that there were antibodies to the common antigens in both heterologous antisera, suggesting circumstantially that these common antigens shared determinants.

\section{Enzyme-linked immunosorbent assay (ELISA)}

The three test antisera were reacted in ELISA with OM complex from each of the ten test strains (Table 1). In only three instances did a heterologous reaction fall below an eighth of the homologous titre, thus indicating strong ELISA reactions of the three antisera with heterologous strains.

Inhibition of ELISA. Inhibition studies 'were performed to investigate the nature of the antigens that reacted in ELISA. Potential inhibitors of the ELISA reactions were $(a)$ homologous untreated (i.e. positive inhibition control), $(b)$ heat treated and $(c)$ periodate treated OM complexes. Antisera were incubated with doubling dilutions of potential inhibitors prior to 

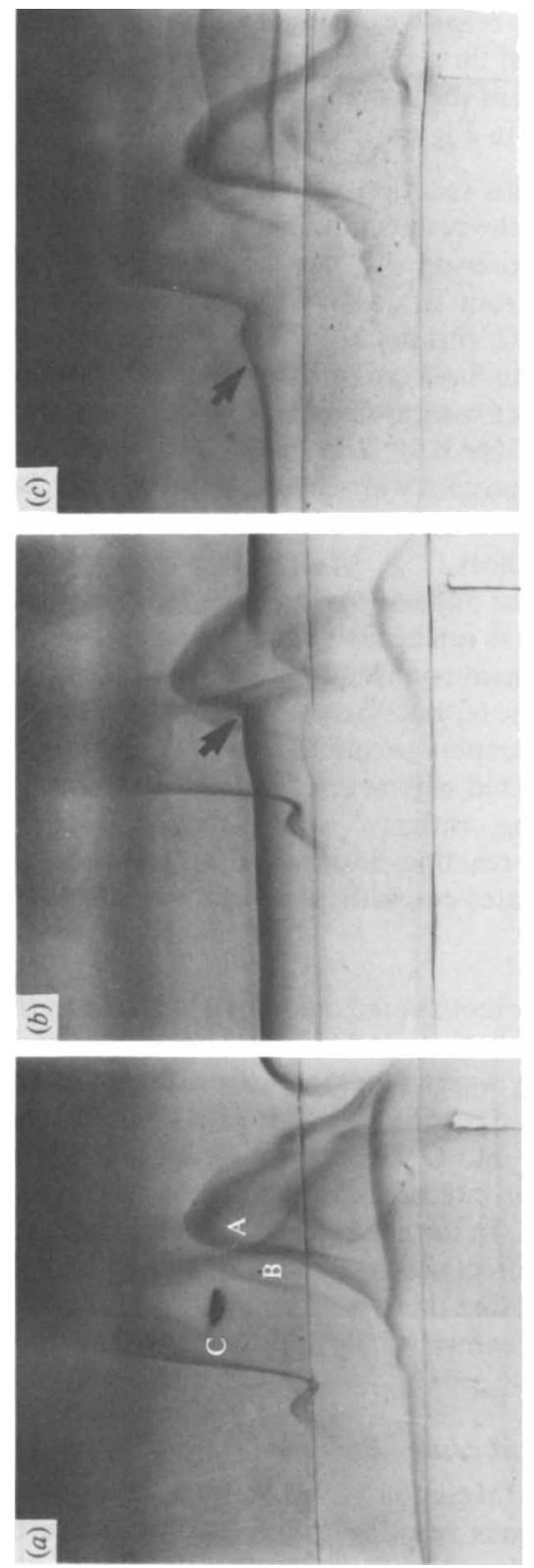

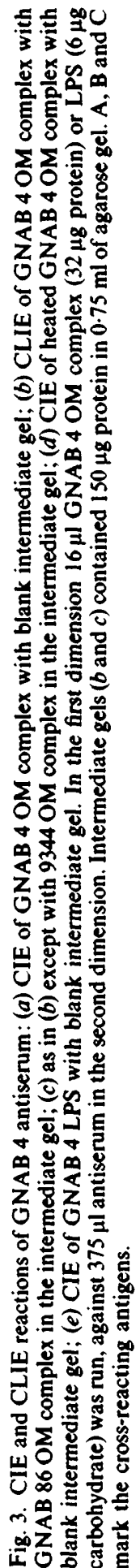




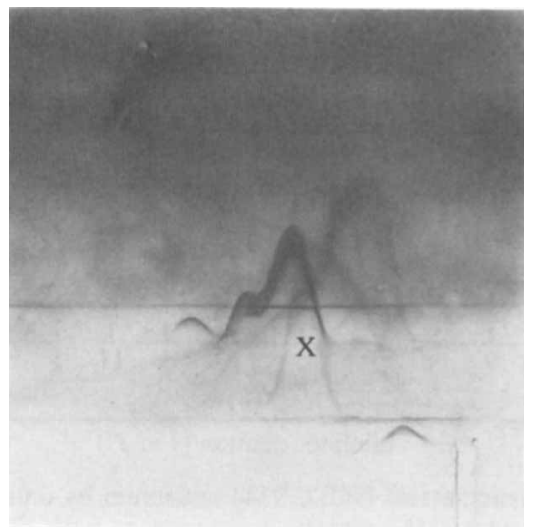

Fig. 4. CIE of GNAB 92 OM complex with NCTC 9344 antiserum in intermediate gel. $16 \mu \mathrm{l}(48 \mu \mathrm{g}$ protein) of GNAB 92 OM complex was run in the first dimension, through an intermediate gel containing $125 \mu 1$ NCTC 9344 antiserum (in $0.75 \mathrm{ml}$ agarose), into $375 \mu \mathrm{l}$ of GNAB 92 antiserum in the second dimension. $\mathrm{X}$ marks the precipitin line which precipitated in the intermediate gel.

Table 1. Reactions of Bacteroides fragilis $O M$ antigens with the three test antisera

The titre is defined as the first dilution of serum with an $A_{405}$ value of less than $1 \cdot 6$. The titre of the homologous reactions is marked $(\mathrm{T})$.

\begin{tabular}{cccc} 
Antigen prepared & \multicolumn{3}{c}{ against antiserum raised against: } \\
\cline { 2 - 4 } from strain no. & NCTC 9344 & GNAB 4 & GNAB 92 \\
NCTC 9344 & $12800(\mathrm{~T})$ & 6400 & 3200 \\
GNAB 4 & 3200 & $25600(\mathrm{~T})$ & 6400 \\
GNAB 85 & 3200 & 6400 & 6400 \\
GNAB 86 & 6400 & 6400 & 6400 \\
GNAB 87 & 3200 & 6400 & 6400 \\
GNAB 88 & 1600 & 1600 & 800 \\
GNAB 90 & 12800 & 3200 & 6400 \\
GNAB 92 & 6400 & 6400 & $25600(\mathrm{~T})$ \\
GNAB 97 & 6400 & 3200 & 3200 \\
GNAB 98 & 12800 & 1600 & 6400
\end{tabular}

addition to the ELISA plate wells. In all experiments, each of the uninhibited reactions (as in Table 1) was included as a control. Absorbance readings in the inhibited reactions were expressed as a percentage of the normal uninhibited ELISA reaction.

Inhibition of NCTC 9344 antiserum reactions. The extent to which heat or periodate treatment reduced the capacity of NCTC 9344 OM complex to inhibit the ELISA reactions of NCTC 9344 antiserum varied, depending on the test antigen. However, three trends occurred in the results and examples of each trend are shown in Fig. 5(a-c). Inhibition of the reaction with GNAB 87 OM complex produced results (Fig. $5 a$ ) representative of those obtained with five other strains (GNAB 4, 85, 87, 90 and 98). The weak inhibition by periodate treated antigens showed that carbohydrates were the major antigens in these reactions. The position of the curve showing inhibition by heated OM complex indicated that both heat resistant and heat labile antigens took part in the reactions. Inhibition of the homologous (NCTC 9344) and GNAB 92 reactions exhibited a second trend (Fig. $5 b$ ): inhibition by heated antigens was similar to that found in the first trend, but periodate treated antigens inhibited to a greater extent than in the first trend. The third trend occurred in the reactions with strains GNAB 88 and GNAB 97 (Fig. 5c) and showed 


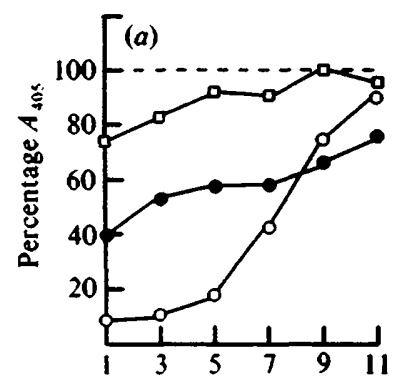

(n)

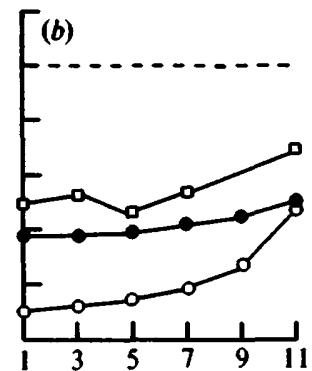

(n)

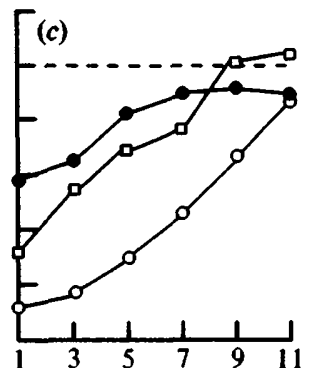

(n)

Inhibitor dilution ( 1 in $2^{n}$ )

Fig. 5. Inhibition of ELISA reactions of NCTC 9344 antiserum by untreated $(O)$, heated $(O)$ and periodate treated $(\square)$ homologous OM complex. (a), reactions with GNAB 87 OM complex; (b), reaction with GNAB 92 OM complex; (c), reaction with GNAB 97 OM complex. $100 \%$ absorbance was that obtained in the control reaction, i.e. no inhibitor added to antiserum.

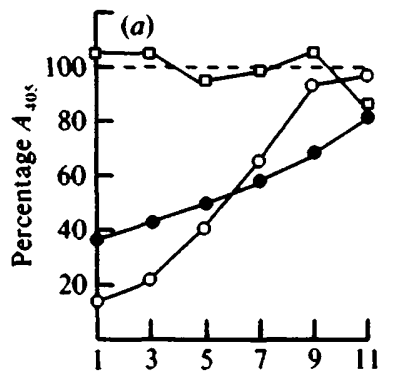

(n)

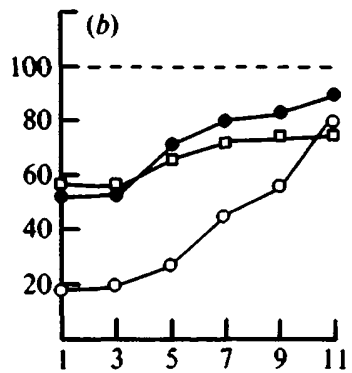

(n)

Inhibitor dilution $\left(1\right.$ in $\left.2^{n}\right)$

Fig. 6. Inhibition of ELISA reactions of GNAB 92 antiserum by untreated $(O)$, heated $(O)$ and periodate treated $(\square)$ homologous OM complex. (a), reactions with GNAB 98 OM complex; (b), reactions with GNAB 88 OM complex.

that heat labile antigens were of greater importance than in the reactions with other strains. The results suggested that reactions showing the second and third trends may have involved important protein antigens as well as carbohydrates.

Inhibition of GNAB 92 antiserum reactions. Two general trends were found in the reactions with GNAB 92 antiserum. The results of the reactions with OM complex from strain GNAB 98 (Fig. $6 a$ ) are an example of the pattern obtained with seven other strains, including the homologous strain. In the GNAB 98 reaction, negligible inhibition occurred with periodate treated inhibitor. In four of the reactions showing this trend, some inhibition occurred at the two lowest inhibitor dilutions, but this became negligible on further dilution. The position of the curve showing inhibition by heated antigens indicated that heat resistant antigens were very important in ELISA, while heat labile antigens were involved to a lesser extent. It is unclear why the heated antigens inhibited ELISA less than untreated antigens at low dilutions and more at high dilutions, but this was a consistent finding in the eight reactions. These results indicated that in eight out of the ten ELISA reactions of GNAB 92 antiserum, carbohydrates were the major antigens.

A second trend occurred in the reactions with strains GNAB 88 (Fig. 6b) and GNAB 97. Heat labile, periodate resistant antigens had a significant role in ELISA, suggesting importance of protein as well as carbohydrate antigens. 


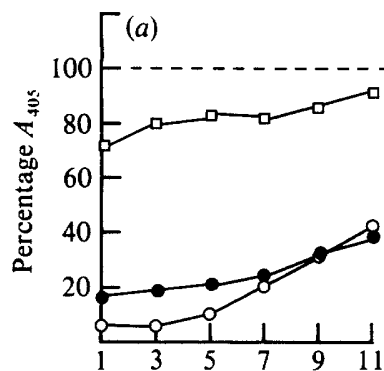

(n)

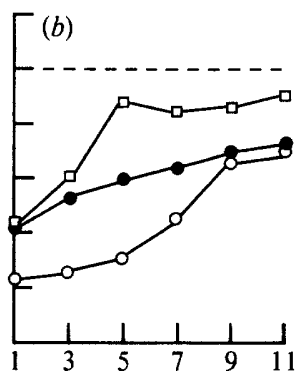

(n)

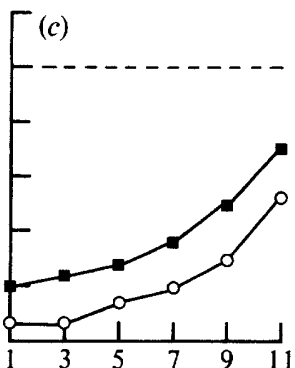

(n)

Inhibitor dilution $\left(1\right.$ in $\left.2^{n}\right)$

Fig. 7. Inhibition of ELISA reactions of GNAB 4 antiserum by untreated $(O)$, heated $(O)$, periodate treated $(\square)$ homologous OM complex and homologous LPS ( $\square)$. (a) and (c), reactions with GNAB 86 OM complex; $(b)$, reaction with GNAB 97 OM complex.

Inhibition of GNAB 4 antiserum reactions. Figure $7(a)$ shows the results obtained in the reactions with OM complex from GNAB 86 . This trend occurred in the reactions with nine strains, including the homologous strain. The strong inhibition by heated antigens indicated that carbohydrates were the predominant antigens in ELISA. Only the reaction with GNAB 97 (Fig. $7 b$ ) differed, but still showed that the most important antigens were carbohydrate.

Inhibition of GNAB 4 antiserum was also carried out with the homologous LPS preparation, with untreated homologous OM complex providing a positive inhibition control. The results obtained in the reactions with strain GNAB 86 (Fig. $7 c$ ) indicated strong inhibition by LPS. A very similar picture was obtained in the reactions with five other strains. In the reactions with the strains GNAB 88, 97 and 98, inhibition by LPS was slightly less than in the GNAB 86 reaction, but was still very significant. Inhibition by LPS was weakest in the homologous reaction.

\section{DISCUSSION}

Throughout this study, an EDTA- $45^{\circ} \mathrm{C}$-mild ultrasonication extract of whole cells was used as the OM complex antigen. From previous work (Poxton \& Ip, 1981; Poxton et al., 1982; Kasper \& Seiler, 1975), we know that this type of preparation represents the outer membrane and associated molecules, including capsule, and contains species-specific antigens.

The techniques of CIE and CLIE have been useful in showing the array of antigens that exist at the cell surface of $B$. fragilis. By these methods, we have detected certain cross-reactions between strains and have examined the antigens present in extracted LPS in relation to OM complex antigens. Most previous studies of the cross-reacting precipitating antigens of $B$. fragilis have used immunodiffusion methods. Immunodiffusion has been applied to the analysis of various extracts of $B$. fragilis and has shown complex patterns of cross-reaction (Abshire et al., 1979; Cherniak et al., 1979; Meisel-Mikolajczyk et al., 1981). It had been concluded that considerable antigenic heterogeneity exists between strains of $B$. fragilis. In the present study, although variations in cross-reacting patterns were observed, two of the test antisera (NCTC 9344 and GNAB 92) each detected heat labile, precipitating antigens common to all strains tested. Such heat labile common precipitating antigens have not previously been reported but Schwan et al. (1981) have demonstrated a heat labile surface antigen associated with non-homogeneous immunofluorescence staining. The reactions of the GNAB 4 antiserum were similar to those observed by previous workers in that no common antigen was demonstrated, heterologous strains cross-reacted with any one of three carbohydrate antigens and no cross-reactions could be demonstrated in some strains. The reason that GNAB 4 antiserum did not detect the single common antigen may have been because this organism has a well defined large capsule which masked, or was dominant over, the common antigen. This is 
supported by the CIE results with GNAB 4 antiserum and homologous LPS and EDTA preparations where the major antigens in both preparations were found to be heat stable and periodate labile. We know from other studies (unpublished results) that the LPS preparation of GNAB 4 contains much high molecular weight capsular polysaccharide.

The nature of the heat labile, common, precipitating antigen(s) detected by the antisera to NCTC 9344 and GNAB 92 strains is not yet fully characterized, but attempts are being made to purify it. Both these strains apparently express this antigen when injected into rabbits. They do not exhibit obvious capsules.

An ELISA has been used to detect largely species-specific reactions of $B$. fragilis antisera with OM complex from heterologous strains (Poxton, 1979). SDS-PAGE of OM complex has shown marked similarities in the polypeptide profiles of different $B$. fragilis strains (Poxton \& Brown, 1979; Kasper \& Seiler, 1975), suggesting that proteins could act as species-specific antigens in ELISA. Alternatively, the species-specific carbohydrate antigen(s) isolated from OM complex (Kasper \& Seiler, 1975; Kasper, 1976) and aqueous phenol extracts (Kasper et al., 1983) may be the major cross-reacting antigen(s) in ELISA. The nature of the antigens reacting in ELISA was investigated in the present study by ELISA inhibition. The results indicated that the predominant cross-reacting antigens were in most cases periodate sensitive and heat resistant and were therefore carbohydrates. The extent to which protein (heat labile, periodate resistant) antigens appeared to be involved in ELISA reactions depended on the individual cross-reacting strain. Although protein antigens were of some significance in ELISA for at least two crossreacting strains (GNAB 88 and GNAB 97), carbohydrate antigens still contributed to these reactions.

There was clearly limited correlation between the CLIE and ELISA results: in the reactions of GNAB 4 antiserum, heat stable carbohydrates were the important cross-reacting antigens in both systems; however, CLIE did not detect cross-reactions with certain strains that crossreacted strongly in ELISA. Heat stable carbohydrates were also predominant in the ELISA reactions with GNAB 92 antiserum, yet CLIE detected only a single, heat labile, cross-reacting antigen with this antiserum. The limited correlation between the two techniques is not unexpected considering the far greater sensitivity of ELISA over CLIE. Also, CLIE only detects precipitating antigens, while ELISA will detect monovalent antigens.

It is not clear which of the carbohydrate components of the OM complex were reacting in ELISA. Inhibition of ELISA reactions of GNAB 4 antiserum with homologous LPS indicated that LPS contained most of the cross-reactive antigens. The nature of the antigens present in the LPS preparations is currently under investigation in this laboratory.

This study was supported by a grant from the Medical Research Council (no. G8106873). G. C. gratefully acknowledges the award of a studentship from the Medical Faculty of the University of Edinburgh.

\section{REFERENCES}

ABShiRe, R. L., DOWELl, V. R. \& LOMBard, G. L. (1979). Serological study of trichloroacetic acid extracts of Bacteroides fragilis. Journal of Clinical Microbiology 9, 274-279.

Cherniax, R., Lombard, G. L. \& Dowell, V. R. (1979). Immunochemical evidence for multiple serotypes of Bacteroides fragilis. Joumal of Clinical Microbiology 9, 699-704.

Dubois, M., Gilles, K. A., Hamilton, J. K., Rebers, P. A. \& SMITH, F. (1956). Colorimetric method for determination of sugars and related substances. Analyrical Chemistry 28, 350-356.

Duerden, B. I., Collee, J. G., Brown, R., Deacon, A. G. \& HolbrooK, W. P. (1980). A scheme for the identification of clinical isolates of Gram-negative anaerobic bacilli by conventional bacteriological tests. Journal of Medical Microbiology 13, 231-245.
Elhag, K. M. \& TABnQCHALI, S. (1978). A study on the surface and somatic antigens of Bacteroides fragilis. Journal of Hygiene 80, 439-449.

FINEGoLd, S. M. (1977). Anaerobic Bacteria in Human Disease. New York: Academic Press.

HorstaD, T. (1975). O-Antigenic specificity of lipopolysaccharide from Bacteroides fragilis ss. fragilis. Acta pathologica et microbiologica scandinavica B83, 477481.

Hofstad, T. (1977). Cross-reactivity of Bacteroides fragilis $\mathrm{O}$-antigens. Acta pathologica et microbiologica scandinavica B85, 9-13.

Hofstad, T. (1981). Precipitating carbohydrate antigens of Bacteroides fragilis NCTC 9343. Acta pathologica et microbiologica scandinavica B89, 215-219.

HoPSTAD, T. (1982). Immunochemical studies of partially degraded lipopolysaccharide from Bacter- 
oides fragilis IPL E323. Acta pathologica, microbiologica et immunologica scandinavica B90, 281-287.

Holbrook, W. P., Duerden, B. I. \& Deacon, A. G. (1977). The classification of Bacteroides melaninogenicus and related species. Journal of Applied Bacteriology 42, 259-273.

KASPER, D. L. (1976). The polysaccharide capsule of Bacteroides fragilis ss. fragilis. Immunochemical and morphologic definition. Journal of Infectious Diseases 133, 79-87.

KASPER, D. L. \& SEILER, M. W. (1975). Immunochemical characterisation of the outer membrane complex of Bacteroides fragilis ss. fragilis. Journal of Infectious Diseases 132, 440-450.

Kasper, D. L., Weintraub, A., Lindberg, A. A. \& LONNGREN, J. (1983). Capsular polysaccharides and lipopolysaccharides from two Bacteroides fragilis strains: chemical and immunochemical characterisation. Journal of Bacteriology 153, 991-997.

KRøLL, J. (1973). Tandem crossed immunoelectrophoresis. Scandinavian Journal of Immunology 2, Suppl. 1, 57-59.

LAMBE, D. W. \& MOROZ, D. A. (1976). Serogrouping of Bacteroides fragilis ss. fragilis by the agglutination test. Journal of Clinical Microbiology 3, 586-592.

Meizel-MikolajczyK, F., Rokosz, A. \& GrzelaKPuCZYNSKA, I. (1981). Serological activity of 'Bacteroides fragilis' endotoxins - strains isolated in Poland. Journal of Applied Bacteriology 51, 399-404.

OnderdonK, A. B., Kasper, D. L., Cisneros, R. L. \& BARTLETT, J. G. (1977). The capsular polysaccharide of Bacteroides fragilis as a virulence factor: comparison of the pathogenic potential of encapsulated and unencapsulated strains. Journal of Infectious Diseases 136, 82-89.
Poxton, I. R. (1979). Serological identification of Bacteroides species by an enzyme-linked immunosorbent assay. Journal of Clinical Pathology 32, 294-298.

Poxton, I. R. \& BRown, R. (1979). Sodium dodecyl sulphate-polyacrylamide gel electrophoresis of cellsurface proteins as an aid to the identification of the Bacteroides fragilis group. Journal of General Microbiology 112, 211-217.

PoXton, I. R. \& BYRNe, M. D. (1981). Immunological analysis of the EDTA-soluble antigens of Clostridium difficile and related species. Journal of General Microbiology 122, 41-46.

Poxton, I. R. \& IP, M. K.-Y. (1981). The cell-surface antigens of Bacteroides vulgatus. Journal of General Microbiology 126, 103-109.

Poxton, I. R., Brown, R. \& Collee, J. G. (1982) Detection of species-specific and cross-reactive cellsurface antigens of Bacteroides species by an indirect enzyme-linked immunosorbent assay. Journal of Medical Microbiology 15, 223-231.

Schwan, A., Danielsson, D. \& Forsum, U. (1982). Demonstration of heat-labile antigen(s) of Bacteroides fragilis associated with non-homogeneous immunofluorescent staining. Acta pathologica, microbiologica et immunologica scandinavica B90, 13-19.

WEEKE, B. (1973). Crossed immunoelectrophoresis. Scandinavian Journal of Immunology 22, Suppl. 1, 4756.

Westrhal, O. \& Luderitz, O. (1954). Chemische Erforschung von Lipopolysacchariden Gram-negativer Bakterien. Angewandte Chemie 66, 407-417.

WREN, M. W. D., BaldWin, A. W. F., Eldon, C. P. \& SANDERSON, P. J. (1977). The anaerobic culture of clinical specimens: a 14-month study. Journal of Medical Microbiology 10, 49-61. 